\title{
The Importance of the Three-Dimensional Image in the Early Diagnosis of Cementoblastoma
}

\author{
Tânia Mara Pimenta Amaral ${ }^{1}$, Cláudia Silami de Magalhães ${ }^{2}$, Allyson Nogueira², \\ Ricardo Alves Mesquita ${ }^{1}$, João Batista de Freitas ${ }^{1}$, Maria Cássia Ferreira Aguiar ${ }^{*}$ \\ ${ }^{1}$ Department of Oral Pathology and Surgery, School of Dentistry, Universidade Federal de Minas Gerais, \\ Belo Horizonte, Brazil \\ ${ }^{2}$ Department of Restorative Dentistry, School of Dentistry, Universidade Federal de Minas Gerais, \\ Belo Horizonte, Brazil \\ Email: *cassiafa@ufmg.br, taniapamaral@uol.com.br
}

Received December 29, 2011; revised January 27, 2012; accepted February 4, 2012

\begin{abstract}
The cementoblastoma is a very rare odontogenic neoplasm characterised by the formation of cementum-like tissue in connection with the root of a tooth. An 18-year-old boy was referred to the Clinic of Oral Pathology of the School of Dentistry, Universidade Federal de Minas Gerais, Brazil, complaining of an irradiating, itching pain from the region of the lower right first molar. He reported that the symptoms began three months prior, with a gradual increase in intensity during this period. The pain episodes were of short duration, with characteristics of toothache originating from the pulp. Upon intra-oral clinical examination, an open bite and slight increase in volume at the buccal alveolar region of the lower right first molar was observed. He also reported palpation and percussion pain symptoms in this region. Electrical, heat and cold pulp vitality tests were performed, and the tooth was vital. Periapical and panoramic radiographs showed radiolucent images with diffuse, poorly defined borders at the mesial root periapex extending to the interradicular region suggesting an inflammatory periapical lesion. In this early presentation, the three-dimensional image was fundamental to the final diagnosis of cementoblastoma.
\end{abstract}

Keywords: Cementoblastoma; Cone Beam CT; Dental Radiography

\section{Case Report}

An 18-year-old boy was referred to the Clinic of Oral Pathology of the School of Dentistry, Universidade Federal de Minas Gerais, Brazil, complaining of an irradiating, itching pain from the region of the lower right first molar. He reported that the symptoms began three months prior, with a gradual increase in intensity during this period. The pain episodes were of short duration, with characterristics of toothache originating from the pulp. There was a gradual increase in pain intensity during this period. His medical history was non-contributory. The physical examination showed a well-developed, well-nourished boy. On extraoral evaluation, his expression and the colour of his face were normal.

The oral examination revealed an open bite and severe pain upon palpation in the mucosa lining the apices of the lower right first molar. The tooth was non-mobile and sensitive to percussion test. A slight bony swelling and tenderness in the right lower posterior buccal gingival tissue were observed. The gingival tissue had a normal aspect (Figure 1(a)). There was no history of previous

\footnotetext{
"Corresponding author.
}

infection associated with this tooth. A periapical and a panoramic radiograph revealed a slight radiolucent area that showed images of ill-defined margins related to the tooth root, associated with a mild bony sclerosis around the periapex of the lower right first molar and with thickening of the periodontal space of the distal root. The trabecular bone at the interradicular region of the lower right first molar tooth was slightly different from the alveolar trabecular bone adjacent to the mesial and distal roots of the lower right first molar. In addition, there was a radiolucent image, which was diffuse and poorly defined, in the furcation area (Figure 1(b)). Based on the clinical and radiologic features of the lesions, a periapical change of inflammatory origin was suspected, but the tooth responded positively to electrical, heat and cold tests of vitality. In order to see major detail of the bone lesion and to obtain the lesion dimensions, cone-beam computed tomography (CBCT) was performed.

The axial, sagittal and coronal planes showed a hyperdense, heterogeneous image, with irregular borders, located at the interradicular region and attached to the mesial and distal roots of the lower right first molar as- 


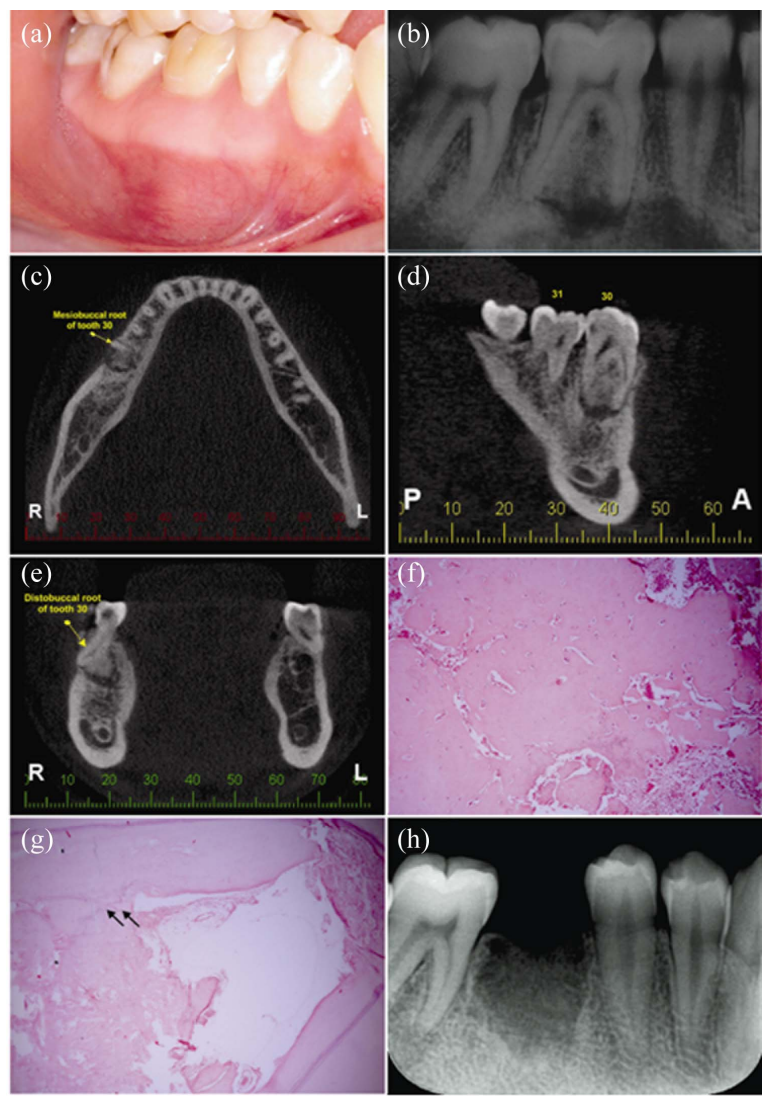

Figure 1. (a) Intraoral view of the left lower quadrant showing discrete swelling and a normal oral mucosa; (b) A slight radiolucent area that showed images of ill-defined margins related to the tooth root. A radiolucent point suggests involvement of the furcation region; (c) A axial image showed a slight cortical expansion and perforation of the cortical buccal bone; (d), (e) The sagittal and coronal images showed a hyperdense, heterogeneous image of irregular borders associated with the hypodense line. The lesion attached to the roots at an early stage of development; (f) Tissue with a compact arrangement, presenting basophilic deposits, interspersed with loose, cellularised connective tissue that was poorly vascularised; (g) Surgical specimen showing cementum-like tissue in connection with the root of the right lower first molar (arrows); (h) Radiological followup. Newly formed trabecular bone in the region of the lower right first molar.

sociated with the hypodense line, represented in the tomographic image. The axial plane showed a slight cortical expansion and perforation of the cortical buccal bone. The CBCT images showed no root resorption (Figures 1(c), (d) and (e)). Osteoblastoma and osteosarcoma were proposed as diagnostic hypotheses. Accordingly, we conducted an incisional biopsy, which showed mineralised tissue with a compact arrangement, presenting basophilic deposits, interspersed with loose, cellularised, poorly vascularised connective tissue (Figure 1(f)). Multinucleated giant cells were observed in proximity to the mineralised tissue. Based on the clinical findings (imaging and histopathology), a diagnosis of benign cementoblastoma was made. Surgical removal of the lesion and the tooth was performed; a peripheral osteotomy was also performed. The piece was sent for histopathologic evaluation, which confirmed the diagnosis (Figure 1(g)). The patient returned for the consultation proservation six months after surgery. A periapical radiograph showed no evidence of recurrence (Figure 1(h)).

\section{Discussion}

Cementoblastoma is a slow-growing, benign odontogenic tumour that arises from cementoblasts. Cementoblastoma is rare and accounts for less than $1 \%$ of all odontogenic tumours [1,2], exhibiting a relatively limited tendency to recur and with unlimited growth potential $[3,4]$. This tumor is characterised by the formation of cementum-like tissue in connection with the root of a tooth and primarily affects young adults in the second and third decades of life $[1,5]$. Some studies have reported that cementoblastoma arises slightly more frequently in males [6], others reported a predominance in females [1], whereas others have found no difference between the sexes [7].

The mandible is the preferential site of occurrence, with the first molar tooth being the most common tooth involved [5,8]. Cementoblastomas associated with primary teeth are extremely rare lesions [3]. Expansion and perforation of the cortex are noted in a higher proportion of patients with recurrent cementoblastoma [9]. Some patients may also complain of associated pain with varying degrees of intensity and occasional paresthesia. The pain is caused by occlusal pressure resulting from extrusion of the tooth caused by pressure from the tumor [10], other patients may be completely asymptomic [11]. The clinical and radiographic features may be varied, causing difficulties in definitive diagnosis and patient management. The diagnosis is rarely made before significant bone expansion occurs [12].

In the present case, the radiographic presentation was not the classic presentation reported by other authors: a radiopaque mass most often fused with the root or roots of a tooth, surrounded and limited peripherally by a radiolucent halo $[1,13]$. On the contrary, the two-dimensional image of the lower right first molar presented an ill-defined radiolucent image associated with mild sclerosis. Based on these images, the diagnosis formulated was an inflammatory cyst or a granuloma and focal cementoosseous dysplasia (FCOD) [6,12]. Inflammatory conditions of the pulp were differentiated by performing tests of pulp vitality, such as the electrical, heat and cold tests. FCOD cannot be distinguished radiographically from an early developing cementoblastoma; both would be associated with vital teeth. The biopsy of FCOD will yield gritty haemorrhagic fragments of tissue because the lesion is difficulty to separate from the adjacent bone [13]. 
A few authors have reported a more radiolucent form of the lesion, and they assumed that it represented an early uncalcified matrix stage [14]. Depending on the stage of maturation, the radiological appearance and clinical interpretation may vary. Occasionally, transitional zones are present between the tumour mass and the tooth substance, so radiographically, opacity gradations at the tumour root structure are hazy and indistinct. Immature lesions are radiolucent, and the differential diagnosis might include an inflammatory cyst, osseous dysplasia, central giant cell lesion or ameloblastoma $[8,15]$.

Radiographically, the cementoblastoma is adhered to the apical or lateral area of the root and appears as a dense radiopaque mass, well defined and circumscribed by a thin radiolucent and uniform halo [9,15]. Radiographic image aspects (such as signs of root resorption, loss of contour of the root, obliteration of the periodontal space) associated with the vitality of the involved tooth are precise, pathognomonic signs $[3,16]$. All of these signs can be seen in two-dimensional images, but that is not what occurred in the present case: root resorption and loss of contour of the root were not visualized. Therefore, in early lesions, when the two-dimensional images do not show defined characteristics, three-dimensional images become necessary. The CBCT images showed no root resorption, however, the CBCT in the axial, coronal and sagittal planes showed, in detail, lesion attachment to the roots at an early stage, discrete jaw expansion and perforation of the cortex, which facilitated diagnosis. These features associated with the histopathological picture allowed the final diagnosis.

In its histological aspects cementoblastoma may sometimes resemble osteoblastoma, or atypical osteosarcoma, and may be difficult to distinguish from these tumours. Osteoblastoma and cementoblastoma are essentially identical histologically; the only distinguishing feature is the attachment of cementoblastoma to the root of the tooth [1,5,17-19]. In cases of osteoblastoma, the symptoms of pain are reduced with the use of a non-narcotic analgesic. The osteoblastoma produces a round, well-demarcated, lytic radiolucent lesion surrounded by a zone of reactive sclerosis [12]. In contrast to the osteoblastoma, the cementoblastoma is an odontogenic tumour that recapitulates cementum deposition, similar to what occurs during formation in the late stages of odontogenesis [12]. Histologically, the cementoblasts in cementoblastoma may be plump with pleomorphic and hyperchromatic nuclei; however, mitotic figures are not seen in cementoblastoma like they are in osteosarcoma [20]. Differentiation of the aforementioned lesions from cementoblastoma requires correlation with radiographic findings, including proximity of the lesion to the roots as well as clinical information, such as age, gender, location and symptoms. Such an approach will allow the clinician to make a de- finitive diagnosis of these lesions [21].

The usual treatment for cementoblastoma is complete surgical excision because the tumour has the potential for unlimited growth. Extraction of the associated tooth is necessary because of the fusion of the lesion to the root cementum, even though the pulp may be vital $[8,9,15]$.

Although this type of early lesion is rarely seen twodimension image, it is important to increase the awareness of this condition among dentists. In conclusion, because of the characteristics of cementoblastoma, the CBCT images are a resource of fundamental importance for evaluation of pathognomonic features related to fusion of the tumour at the root of the tooth in the early stages of development.

\section{Acknowledgements}

This study was supported by grants from the National Council for Scientific and Technological DevelopmentCNPq 302711/2010-4, FAPEMIG PPM-00516-11 and Pró-Reitoria de Pesquisa da Universidade Federal de Minas Gerais. Dr. Aguiar, and Dr. Mesquita are research fellows of CNPq.

\section{REFERENCES}

[1] M. Ulmansky, E. Hjørting-Hansen, F. Praetorius and M. F. Haque, "Benign Cementoblastoma. A Review and Five New Cases,” Oral Surgery, Oral Medicine, Oral Pathology, Oral Radiology \& Endodontics, Vol. 77, No. 1, 1994, pp. 48-55.

[2] I. Van der Waal, “Cementoblastoma,” In: L. Barnes, J. W. Eveson, P. Reichart and D. Sidransky, Eds., World Health Organization Classification of Tumours, Pathology \& Genetics: Head and Neck Tumours, Lyon, IARC Press, 2005.

[3] K. Lemberg, J. Hagström, J. Rihtniemi and K. Soikkonen, "Benign Cementoblastoma in a Primary Lower Molar, a Rarity,” Dentomaxillofacial Radiology, Vol. 36, No. 6, 2007, pp. 364-366. doi:10.1259/dmfr/58249657

[4] A. P. Vieira, J. M. Meneses Jr. and R. L. Maia, "Cementoblastoma Related to a Primary Tooth: A Case Report," Journal of Oral Pathology \& Medicine, Vol. 36, No. 2, 2007, pp. 117-119. doi:10.1111/j.1600-0714.2007.00465.x

[5] M. Sumer, K. Gunduz, A. P. Sumer and O. Gunhan, "Benign Cementoblastoma: A Case Report,” Medicina Oral, Patología Oral y Cirugía Bucal, Vol. 11, No. 6, 2006, pp. E483-E485.

[6] A. G. Farman, W. W. Köhler, C. J. Nortjé and C. W. Van Wyk, "Cementoblastoma: Report of Case," Journal of Oral Surgery, Vol. 37, No. 3, 1979, pp. 198-203.

[7] D. S. MacDonald-Jankowski and P. C. Wu, "Cementoblastoma in Hong Kong Chinese. A Report of Four Cases," Oral Surgery, Oral Medicine, Oral Pathology, Vol. 73, No. 6, 1992, pp. 760-764.

[8] P. Infante-Cossio, J. M. Hernandez-Guisado, M. Acosta- 
Feria and A. Carranza-Carranza, "Cementoblastoma Involving the Maxillary Sinus,” British Journal of Oral and Maxillofacial Surgery, Vol. 46, No. 3, 2008, pp. 234-236. doi:10.1016/j.bjoms.2007.03.009

[9] R. B. Brannon, C. B. Fowler, W. M. Carpenter and R. L. Corio, "Cementoblastoma: An Innocuous Neoplasm? A Clinicopathologic Study of 44 Cases and Review of the Literature with Special Emphasis on Recurrence," Oral Surgery, Oral Medicine, Oral Pathology, Oral Radiology \& Endodontics, Vol. 93, No. 3, 2002, pp. 311-320. doi:10.1067/moe.2002.121993

[10] A. M. Abrams, J. W. Kirby and R. J. Melrose, “Cementoblastoma. A Clinical-Pathologic Study of Seven New Cases," Oral Surgery, Oral Medicine, Oral Pathology, Oral Radiology \& Endodontics, Vol. 38, No. 3, 1974, pp. 394-403.

[11] J. T. Biggs and F. W. Benenati, "Surgically Treating a Benign Cementoblastoma While Retaining the Involved Tooth,” Journal of the American Dental Association, Vol. 126, No. 9, 1995, pp. 1288-1290.

[12] E. J. Cundiff 2nd, "Developing Cementoblastoma: Case Report and Update of Differential Diagnosis,” Quintessence International, Vol. 31, No. 3, 2000, pp. 191-195.

[13] L. Napier Souza, S. Monteiro Lima Júnior, F. J. Garcia Santos Pimenta, A. C. Rodrigues Antunes Souza and R. Santiago Gomez, "Atypical Hypercementosis versus Cementoblastoma,” Dentomaxillofacial Radiology, Vol. 33, No, 4, 2004, pp. 267-270. doi:10.1259/dmfr/30077628

[14] J. C. Gingell, M. Lunin, T. Beckerman and B. A. Levy, "Benign Cementoblastoma," Journal of Oral Medicine, Vol. 39, No. 1, 1984, pp. 8-11.

[15] J. S. Jelic, M. J. Loftus, A. S. Miller and D. B. Cleveland,
"Benign Cementoblastoma: Report of an Unusual Case and Analysis of 14 Additional Cases," Journal of Oral and Maxillofacial Surgery, Vol. 51, No. 9, 1993, pp. 1033-1037. doi:10.1016/S0278-2391(10)80051-5

[16] H. Zaitoun, O. Kujan and P. Sloan, “An Unusual Recurrent Cementoblastoma Associated with a Developing Lower Second Molar Tooth: A Case Report,” Journal of Oral and Maxillofacial Surgery, Vol. 65, No. 10, 2007, pp. 2080-2082. doi:10.1016/j.joms.2006.06.288

[17] K. Ohki, H. Kumamoto, Y. Nitta, H. Nagasaka, H. Kawamura and K. Ooya, "Benign Cementoblastoma Involving Multiple Maxillary Teeth: Report of a Case with a Review of the Literature,” Oral Surgery, Oral Medicine, Oral Pathology, Oral Radiology \& Endodontics, Vol. 97, No. 1, 2004, pp. 53-58. doi:10.1016/j.tripleo.2003.08.012

[18] E. Hirai, K. Yamamoto, T. Kounoe, Y. Kondo, H. Yonemasu and H. Kurokawa, "Benign Cementoblastoma of the Anterior Maxilla," Journal of Oral and Maxillofacial Surgery, Vol. 68, No. 3, 2010, pp. 671-674. doi:10.1016/j.joms.2009.03.060

[19] A. R. Huber and G. S. Folk, “Cementoblastoma,” Head \& Neck Pathology, Vol. 3, No. 2, 2009, pp. 133-135. doi:10.1007/s12105-008-0099-5

[20] P. J. Slootweg, “Cementoblastoma and Osteoblastoma: A Comparison of Histologic Features," Journal of Oral Pathology \& Medicine, Vol. 21, No. 9, 1992, pp. 385-389. doi:10.1111/j.1600-0714.1992.tb01024.X

[21] E. Bilodeau, B. Collins, B. Costello and A. Potluri, "Case Report: A Pediatric Case of Cementoblastoma with Histologic and Radiographic Features of an Osteoblastoma and Osteosarcoma," Head \& Neck Pathology, Vol. 4, No. 4, 2010, pp. 324-328. doi:10.1007/s12105-010-0205-3 